\title{
A note on maximal singular integrals with rough kernels
}

Xiao Zhang ${ }^{1}$ and Feng Liu ${ }^{2 *}$ (i)

"Correspondence:

liufeng860314@163.com

${ }^{2}$ College of Mathematics and

System Science, Shandong

University of Science and

Technology, Qingdao, 266590, P.R.

China

Full list of author information is

available at the end of the article

\begin{abstract}
In this note we study the maximal singular integral operators associated with a homogeneous mapping with rough kernels as well as the corresponding maximal operators. The boundedness and continuity on the Lebesgue spaces, Triebel-Lizorkin spaces, and Besov spaces are established for the above operators with rough kernels in $H^{1}\left(\mathrm{~S}^{n-1}\right)$, which complement some recent developments related to rough maximal singular integrals.
\end{abstract}

MSC: Primary 42B20; secondary 42B25

Keywords: Maximal singular integral; Maximal operator; $H^{1}\left(\mathrm{~S}^{n-1}\right)$; Homogeneous mapping; Triebel-Lizorkin space

\section{Introduction}

During the last several years, a considerable amount of attention has been given to investigate the boundedness for various kinds of integral operators on Triebel-Lizorkin spaces. For examples, see $[1,4,5]$ for singular integrals, $[15,17,26,27]$ for Marcinkiewicz integrals, $[16,27]$ for Littlewood-Paley functions, $[14,18,20]$ for maximal functions, and $[21,22]$ for maximal singular integrals. The main purpose of this paper is to prove the boundedness and continuity of the maximal singular integral and maximal operators related to homogeneous mappings on Triebel-Lizorkin spaces when their kernels are given by function $\Omega$ in the Hardy space $H^{1}\left(\mathrm{~S}^{n-1}\right)$. Let $n, d \geq 2$ and $m=\left(m_{1}, \ldots, m_{d}\right) \in \mathbb{R}^{d}$. We say that $\Phi: \mathbb{R}^{n} \rightarrow \mathbb{R}^{d}$ is a (nonisotropic) homogeneous mapping of degree $m$ if

$$
\Phi(t y)=\delta_{t}(\Phi(y))
$$

holds for all $t>0$ and $y \in \mathbb{R}^{d}$. Here, $\left\{\delta_{t}\right\}_{t>0}$ is a family of dilations on $\mathbb{R}^{d}$ defined by

$$
\delta_{t}\left(x_{1}, \ldots, x_{d}\right)=\left(t^{m_{1}} x_{1}, \ldots, t^{m_{d}} x_{d}\right) .
$$

Before stating our main results, let us recall some pertinent notations, definitions, and background. Let $S^{n-1}$ be the unit sphere in $\mathbb{R}^{n}$ equipped with the induced Lebesgue mea-

(c) The Author(s) 2020. This article is licensed under a Creative Commons Attribution 4.0 International License, which permits use, sharing, adaptation, distribution and reproduction in any medium or format, as long as you give appropriate credit to the original author(s) and the source, provide a link to the Creative Commons licence, and indicate if changes were made. The images or other third party material in this article are included in the article's Creative Commons licence, unless indicated otherwise in a credit line to the material. If material is not included in the article's Creative Commons licence and your intended use is not permitted by statutory regulation or exceeds the permitted use, you will need to obtain permission directly from the copyright holder. To view a copy of this licence, visit http://creativecommons.org/licenses/by/4.0/. 
sure $d \sigma$. Let $\Omega$ be integrable over $S^{n-1}$ and satisfy

$$
\int_{S^{n-1}} \Omega(u) d \sigma(u)=0
$$

For a suitable mapping $\Phi: \mathbb{R}^{n} \rightarrow \mathbb{R}^{d}$, we define the singular integral operator $T_{\Omega, \Phi}$ by

$$
T_{\Omega, \Phi} f(x):=\text { p.v. } \int_{\mathbb{R}^{n}} f(x-\Phi(y)) \frac{\Omega(y /|y|)}{|y|^{n}} d y,
$$

where $f \in \mathcal{S}\left(\mathbb{R}^{d}\right)$ (the Schwartz class on $\mathbb{R}^{d}$ ). When $n=d$ and $\Phi(y)=y$, the operator $T_{\Omega, \Phi}$ reduces to the classical Calderón-Zygmund singular integral operator, which is denoted by $T_{\Omega}$. In their fundamental work on singular integrals, Calderón and Zygmund [3] first proved that $T_{\Omega}$ is bounded on $L^{p}\left(\mathbb{R}^{n}\right)$ for $1<p<\infty$ if $\Omega \in L \log L\left(S^{n-1}\right)$. The same conclusion was obtained independently by Coifman and Weiss [7] and Connett [8] under the less restrictive condition that $\Omega \in H^{1}\left(\mathrm{~S}^{n-1}\right)$. Here, $H^{1}\left(\mathrm{~S}^{n-1}\right)$ denotes the Hardy space on the unit sphere and contains $L \log L\left(\mathrm{~S}^{n-1}\right)$ as a proper subspace. The above results were later extended to singular Radon transforms by many authors (see $[2,6,9,10])$. In particular, Cheng [6] proved the following result.

Theorem A ([6]) Let $\Phi=\left(\Phi_{1}, \ldots, \Phi_{d}\right)$ be a homogeneous mapping of degree $m=\left(m_{1}, \ldots\right.$, $\left.m_{d}\right)$ with each $m_{i} \neq 0$. Assume that $\Omega \in H^{1}\left(S^{n-1}\right)$ satisfies $(1.1)$ and $\left.\Phi\right|_{S^{n-1}}$ is real-analytic. Then, for $1<p<\infty$, there exists a positive constant $C_{p}$ such that

$$
\left\|T_{\Omega, \Phi} f\right\|_{L^{p}\left(\mathbb{R}^{d}\right)} \leq C_{p}\|f\|_{L^{p}\left(\mathbb{R}^{d}\right)}
$$

In this paper, we study the maximal singular integrals and maximal operators related to homogeneous mappings. Let $\Omega, \Phi$ be given as in (1.2). The maximal singular integral operator $T_{\Omega, \Phi}^{*}$ and the maximal operator $\mathrm{M}_{\Omega, \Phi}$ are defined by

$$
\begin{aligned}
& T_{\Omega, \Phi}^{*} f(x):=\sup _{\epsilon>0}\left|\int_{|y|>\epsilon} f(x-\Phi(y)) \frac{\Omega(y /|y|)}{|y|^{n}} d y\right|, \\
& \mathrm{M}_{\Omega, \Phi} f(x)=\sup _{t>0} \frac{1}{t^{n}} \int_{|y|<t}|f(x-\Phi(y))||\Omega(y /|y|) h(|y|)| d y .
\end{aligned}
$$

For the sake of simplification, we denote $T_{\Omega, \Phi}^{*}=T_{\Omega}$ and $\mathrm{M}_{\Omega, \Phi}=\mathrm{M}_{\Omega}$ when $n=d$ and $\Phi(y)=y$. Particularly, when $\Phi=\mathcal{P}$ is a real-valued polynomial mapping from $\mathbb{R}^{n}$ to $\mathbb{R}^{d}$, we denote $T_{\Omega, \Phi}^{*}=T_{\Omega, \mathcal{P}}^{*}$ and $\mathrm{M}_{\Omega, \Phi}=\mathrm{M}_{\Omega, \mathcal{P}}$. In 1997, Fan and Pan [10] proved that $T_{\Omega, \mathcal{P}}^{*}$ is bounded on $L^{p}\left(\mathbb{R}^{d}\right)$ for $1<p<\infty$, provided that $\Omega \in H^{1}\left(\mathrm{~S}^{n-1}\right)$. It follows from a theorem of Stein and Wainger that $\mathrm{M}_{\Omega, \mathcal{P}}$ is bounded on $L^{p}\left(\mathbb{R}^{d}\right)$ for $1<p \leq \infty$ if $\Omega \in L^{1}\left(\mathrm{~S}^{n-1}\right)$. Other relevant results on the $L^{p}$ bounds for $\mathrm{M}_{\Omega, \mathcal{P}}$ can be found in [2, 24].

Based on the above, a natural question is the following:

Question A Are the operators $T_{\Omega, \Phi}^{*}$ and $\mathrm{M}_{\Omega, \Phi}$ bounded on $L^{p}\left(\mathbb{R}^{d}\right)$ for all $1<p<\infty$ if $\Omega$ and $\Phi$ are given as in Theorem $\mathrm{A}$ ?

This question can be addressed by the following theorem. 
Theorem 1.1 Let $\Phi=\left(\Phi_{1}, \ldots, \Phi_{d}\right)$ be a homogeneous mapping of degree $m=\left(m_{1}, \ldots, m_{d}\right)$ with each $m_{i} \neq 0$ and $\left.\Phi\right|_{S^{n-1}}$ real-analytic. Assume that $\Omega \in H^{1}\left(S^{n-1}\right)$ satisfies (1.1). Then, for $1<p<\infty$, there exists a positive constant $C_{p}$ such that

$$
\begin{aligned}
& \left\|T_{\Omega, \Phi}^{*} f\right\|_{L^{p}\left(\mathbb{R}^{d}\right)} \leq C_{p}\|\Omega\|_{H^{1}\left(S^{n-1}\right)}\|f\|_{L^{p}\left(\mathbb{R}^{d}\right)}, \\
& \left\|\mathrm{M}_{\Omega, \Phi} f\right\|_{L^{p}\left(\mathbb{R}^{d}\right)} \leq C_{p}\|\Omega\|_{H^{1}\left(\mathrm{~S}^{n-1}\right)}\|f\|_{L^{p}\left(\mathbb{R}^{d}\right)} .
\end{aligned}
$$

Here, the above constant $C>0$ is independent of $\Omega$.

Remark 1.1 We point out the following remarks, which are useful in comprehending Theorem 1.1.

(i) Theorem 1.1 is expectant. Actually, Theorem 1.1 can be proved by applying the arguments similar to those used in deriving [6, Theorem 1] and [10, Lemma 6.3]. However, our main arguments in proving Theorem 1.1 are different from those of [6, Theorem 1].

(ii) Theorem 1.1 is new. In a very recent paper [19], the authors have established the $L^{p}(1<p<\infty)$ bounds for the singular integral operator and maximal singular integral operator related to homogeneous mappings when their integral kernels are given by the unit sphere kernel in $H^{1}\left(\mathrm{~S}^{n-1}\right)$ and the weak size radial kernel, which contradicts the main result of [13].

On the other hand, the boundedness properties of maximal singular integral operator and maximal operator in Triebel-Lizorkin spaces have also received some attention of many authors. Let $\mathcal{S}^{\prime}\left(\mathbb{R}^{d}\right)$ be the tempered distribution class on $\mathbb{R}^{d}$. For $\alpha \in \mathbb{R}$ and $0<$ $p, q \leq \infty(p \neq \infty)$, the homogeneous Triebel-Lizorkin spaces $\dot{F}_{\alpha}^{p, q}\left(\mathbb{R}^{d}\right)$ are defined by

$$
\dot{F}_{\alpha}^{p, q}\left(\mathbb{R}^{d}\right):=\left\{f \in \mathcal{S}^{\prime}\left(\mathbb{R}^{d}\right):\|f\|_{\dot{F}_{\alpha}^{p, q}\left(\mathbb{R}^{d}\right)}=\left\|\left(\sum_{i \in \mathbb{Z}} 2^{-i \alpha q}\left|\Psi_{i} * f\right|^{q}\right)^{1 / q}\right\|_{L^{p}\left(\mathbb{R}^{d}\right)}<\infty\right\}
$$

where $\widehat{\Psi}_{i}(\xi)=\phi\left(2^{i} \xi\right)$ for $i \in \mathbb{Z}$ and $\phi \in \mathcal{C}_{c}^{\infty}\left(\mathbb{R}^{d}\right)$ satisfies the conditions: $0 \leq \phi(x) \leq 1$; $\operatorname{supp}(\phi) \subset\{x: 1 / 2 \leq|x| \leq 2\} ; \phi(x)>c>0$ if $3 / 5 \leq|x| \leq 5 / 3$. The inhomogeneous version of Triebel-Lizorkin spaces, which is denoted by $F_{\alpha}^{p, q}\left(\mathbb{R}^{d}\right)$, is obtained by adding the term $\|\Theta * f\|_{L^{p}\left(\mathbb{R}^{d}\right)}$ to the right-hand side of (1.3) with $\sum_{i \in \mathbb{Z}}$ replaced by $\sum_{i \geq 1}$, where $\Theta \in \mathcal{S}\left(\mathbb{R}^{d}\right)$, $\operatorname{supp}(\hat{\Theta}) \subset\{\xi:|\xi| \leq 2\}, \hat{\Theta}(x)>c>0$ if $|x| \leq 5 / 3$. It is well known that the following are valid (see $[11,25]$ for more details):

$$
\begin{aligned}
& \dot{F}_{0}^{p, 2}\left(\mathbb{R}^{d}\right)=L^{p}\left(\mathbb{R}^{d}\right) \quad \text { for } 1<p<\infty ; \\
& F_{\alpha}^{p, q}\left(\mathbb{R}^{d}\right) \sim \dot{F}_{\alpha}^{p, q}\left(\mathbb{R}^{d}\right) \cap L^{p}\left(\mathbb{R}^{d}\right) \quad \text { and } \\
& \left.\|f\|_{F_{\alpha}^{p, q}\left(\mathbb{R}^{d}\right)} \sim\|f\|_{\dot{F}_{\alpha}^{p, q}} \mathbb{R}^{d}\right) \\
& +\|f\|_{L^{p}\left(\mathbb{R}^{d}\right)} \quad \text { for } \alpha>0 .
\end{aligned}
$$

Recently, Liu et al. [21, 22] have established the bounds for $T_{\Omega, \mathcal{P}}^{*}$ and $\mathrm{M}_{\Omega, \mathcal{P}}$ on TriebelLizorkin spaces when $\Omega \in L \log L\left(\mathrm{~S}^{n-1}\right)$ or $\Omega \in \mathcal{F}_{\beta}\left(\mathrm{S}^{n-1}\right)$ (the Grafakos-Stefanov function class (see [12])). It should be pointed out that the following relationships are valid:

$$
\begin{aligned}
& L(\log L)^{\alpha_{1}}\left(\mathrm{~S}^{n-1}\right) \subsetneq L(\log L)^{\alpha_{2}}\left(\mathrm{~S}^{n-1}\right) \quad \text { for } 0<\alpha_{2}<\alpha_{1} \\
& L(\log L)^{\alpha}\left(\mathrm{S}^{n-1}\right) \subsetneq H^{1}\left(\mathrm{~S}^{n-1}\right) \subsetneq L^{1}\left(\mathrm{~S}^{n-1}\right) \quad \text { for } \alpha \geq 1 ;
\end{aligned}
$$




$$
\begin{aligned}
& L(\log L)^{\alpha}\left(\mathrm{S}^{n-1}\right) \nsubseteq H^{1}\left(\mathrm{~S}^{n-1}\right) \nsubseteq L(\log L)^{\alpha}\left(\mathrm{S}^{n-1}\right) \quad \text { for } 0<\alpha<1 ; \\
& \bigcap_{\beta>1} \mathcal{F}_{\beta}\left(\mathrm{S}^{n-1}\right) \nsubseteq H^{1}\left(\mathrm{~S}^{n-1}\right) \nsubseteq \bigcup_{\beta>1} \mathcal{F}_{\beta}\left(\mathrm{S}^{n-1}\right) ; \\
& \bigcap_{\beta>1} \mathcal{F}_{\beta}\left(\mathrm{S}^{n-1}\right) \nsubseteq L \log L\left(\mathrm{~S}^{n-1}\right) .
\end{aligned}
$$

As far as I know the bounds for $T_{\Omega, \mathcal{P}}^{*}$ and $\mathrm{M}_{\Omega, \mathcal{P}}$ on the Triebel-Lizorkin spaces and Besov spaces are unknown under the condition that $\Omega \in H^{1}\left(\mathrm{~S}^{n-1}\right)$, even in the special case $n=d$ and $\mathcal{P}(y)=y$.

A natural question, which arises from the above, is the following:

Question 1.1 Are the operators $T_{\Omega}^{*}$ and $\mathrm{M}_{\Omega}$ bounded on the Triebel-Lizorkin spaces if $\Omega \in H^{1}\left(\mathrm{~S}^{n-1}\right)$ ?

Question 1.1 is the main motivation for this work. In this paper we give an affirmative answer to the above question by treating more general operators. Our main result can be stated as follows.

Theorem 1.2 Let $\Phi=\left(\Phi_{1}, \ldots, \Phi_{d}\right)$ be a homogeneous mapping of degree $m=\left(m_{1}, \ldots, m_{d}\right)$ with each $m_{i} \in \mathbb{N} \backslash\{0\}$ and $\left.\Phi\right|_{S^{n-1}}$ real-analytic. Assume that $\Omega \in H^{1}\left(\mathrm{~S}^{n-1}\right)$ satisfies (1.1). Then

(i) For any $\alpha \in(0,1)$ and $1<p, q<\infty$, there exists a constant $C>0$ such that

$$
\left\|T_{\Omega, \Phi}^{*} f\right\|_{\dot{F}_{\alpha}^{p, q}\left(\mathbb{R}^{d}\right)} \leq C\|\Omega\|_{H^{1}\left(S^{n-1}\right)}\|f\|_{\dot{F}_{\alpha}^{p, q}\left(\mathbb{R}^{d}\right)},
$$

where $C>0$ is independent of $\Omega$.

(ii) $T_{\Omega, \Phi}^{*}$ is continuous from $F_{\alpha}^{p, q}\left(\mathbb{R}^{d}\right)$ to $\dot{F}_{\alpha}^{p, q}\left(\mathbb{R}^{d}\right)$ for all $\alpha \in(0,1)$ and $1<p, q<\infty$.

The same conclusions hold for $\mathrm{M}_{\Omega, \Phi}$.

The rest of this section is to present the bounds and continuity for $T_{\Omega, \Phi}^{*}$ and $\mathrm{M}_{\Omega, \Phi}$ on Besov spaces. For $\alpha \in \mathbb{R}$ and $0<p, q \leq \infty(p \neq \infty)$, the homogeneous Besov spaces $\dot{B}_{\alpha}^{p, q}\left(\mathbb{R}^{d}\right)$ are defined by

$$
\dot{B}_{\alpha}^{p, q}\left(\mathbb{R}^{d}\right):=\left\{f \in \mathcal{S}^{\prime}\left(\mathbb{R}^{d}\right):\|f\|_{\dot{B}_{\alpha}^{p, q}\left(\mathbb{R}^{d}\right)}=\left(\sum_{i \in \mathbb{Z}} 2^{-i \alpha q}\left\|\Psi_{i} * f\right\|_{L^{p}\left(\mathbb{R}^{d}\right)}^{q}\right)^{1 / q}<\infty\right\}
$$

where $\widehat{\Psi}_{i}$ is given as in (1.3). The inhomogeneous version of Besov spaces $B_{\alpha}^{p, q}\left(\mathbb{R}^{d}\right)$ is obtained by adding the term $\|\Theta * f\|_{L^{p}\left(\mathbb{R}^{d}\right)}$ to the right-hand side of (1.5) with $\sum_{i \in \mathbb{Z}}$ replaced by $\sum_{i \geq 1}$, where $\Theta$ is given as in the definition of $F_{\alpha}^{p, q}\left(\mathbb{R}^{d}\right)$. The following property is well known (see [11, 25] for more details):

$$
\begin{aligned}
& B_{\alpha}^{p, q}\left(\mathbb{R}^{d}\right) \sim \dot{B}_{\alpha}^{p, q}\left(\mathbb{R}^{d}\right) \cap L^{p}\left(\mathbb{R}^{d}\right) \quad \text { and } \\
& \|f\|_{B_{\alpha}^{p, q}\left(\mathbb{R}^{d}\right)} \sim\|f\|_{\dot{B}_{\alpha}^{p, q}\left(\mathbb{R}^{d}\right)}+\|f\|_{L^{p}\left(\mathbb{R}^{d}\right)} \quad \text { for } \alpha>0 .
\end{aligned}
$$

Recently, Liu and Wu [20] established a criterion on the boundedness and continuity of a class of operators on Besov spaces, which is listed as follows. 
Proposition 1.1 ([20]) Let $T$ be a sublinear operator and be bounded on $L^{p}\left(\mathbb{R}^{d}\right)$ for some $p \in(1, \infty)$. Assume that

$$
\left|\Delta_{\zeta}(T f)(x)\right| \leq\left|T\left(\Delta_{\zeta} f\right)(x)\right|
$$

for any $x, \zeta \in \mathbb{R}^{d}$, where $\Delta_{\zeta} f(x)=f(x+\zeta)-f(x)$ for all $x, \zeta \in \mathbb{R}^{d}$. Then $T$ is bounded on $\dot{B}_{\alpha}^{p, q}\left(\mathbb{R}^{d}\right)$ for any $\alpha \in(0,1)$ and $q \in(1, \infty)$. Specially, if $T$ satisfies the following:

$$
|T f-T g| \leq|T(f-g)|
$$

for arbitrary function $f, g$ defined on $\mathbb{R}^{d}$, then $T$ is continuous from $B_{\alpha}^{p, q}\left(\mathbb{R}^{d}\right)$ to $\dot{B}_{\alpha}^{p, q}\left(\mathbb{R}^{d}\right)$ for any $\alpha \in(0,1)$ and $q \in(1, \infty)$.

It is easy to check that both the operators $T_{\Omega, \Phi}^{*}$ and $M_{\Omega, \Phi}$ satisfy conditions (1.7) and (1.8). This together with Theorem 1.1 and Proposition 1.1 implies the following theorem.

Theorem 1.3 Let $\Omega, \Phi$ be given as in Theorem 1.1. Then:

(i) For any $\alpha \in(0,1)$ and $1<p, q<\infty$, there exists a constant $C>0$ such that

$$
\left\|T_{\Omega, \Phi}^{*} f\right\|_{\dot{B}_{\alpha}^{p, q}\left(\mathbb{R}^{d}\right)} \leq C\|\Omega\|_{H^{1}\left(S^{n-1}\right)}\|f\|_{\dot{B}_{\alpha}^{p, q}\left(\mathbb{R}^{d}\right)},
$$

where $C>0$ is independent of $\Omega$.

(ii) $T_{\Omega, \Phi}^{*}$ is continuous from $B_{\alpha}^{p, q}\left(\mathbb{R}^{d}\right)$ to $\dot{B}_{\alpha}^{p, q}\left(\mathbb{R}^{d}\right)$ for all $\alpha \in(0,1)$ and $1<p, q<\infty$.

The same conclusions hold for $\mathrm{M}_{\Omega, \Phi}$.

By (1.4), (1.6), and Theorems 1.1-1.3, we can get immediately the following result.

Theorem 1.4 Let $\Omega$, $\Phi$ be given as in Theorem 1.2. Then both the operators $T_{\Omega, \Phi}^{*}$ and $\mathrm{M}_{\Omega, \Phi}$ are bounded and continuous on $F_{\alpha}^{p, q}\left(\mathbb{R}^{d}\right)$ and $B_{\alpha}^{p, q}\left(\mathbb{R}^{d}\right)$ for $\alpha \in(0,1)$ and $1<p, q<\infty$.

Remark 1.2 Theorems 1.2-1.4 are new, even in the special case $n=d$ and $\Phi(y)=y$.

The paper is organized as follows. Sect. 2 contains the atomic decomposition of Hardy space and some estimates of oscillatory integrals, which play key roles in the estimates of Fourier transforms on some measures. In Sect. 3, we prove Theorem 1.1 after presenting a general criterion on the $L^{p}$ bounds of the convolution operators (see Lemma 3.1). The proof of Theorem 1.2 is given in Sect. 4. It should be pointed out that the proof of the boundedness (resp., continuity) part in Theorem 1.2 is greatly motivated by the idea in [21] (resp., [22]).

Throughout the paper, the letter $C$ or $c$, sometimes with certain parameters, stands for positive constants not necessarily the same one at each occurrence, but are independent of the essential variables. For notational convenience, we set $\exp (i t)=e^{i t}$ for any $t \in \mathbb{R}$.

\section{Preliminary definitions and lemmas}

We start with the definition of Hardy space on $\mathrm{S}^{n-1}$ and its atomic decomposition. Recall that the Poisson kernel on $S^{n-1}$ is defined by $P_{r w}(\theta)=\frac{1-r^{2}}{|r w-\theta|^{n}}$ for $0 \leq r<1$ and $\theta, w \in S^{n-1}$. 
The Hardy space $H^{1}\left(\mathrm{~S}^{n-1}\right)$ is the set of all $L^{1}\left(\mathrm{~S}^{n-1}\right)$ functions which satisfy

$$
\|\Omega\|_{H^{1}\left(S^{n-1}\right)}=\int_{S^{n-1}} \sup _{0 \leq r<1}\left|\int_{S^{n-1}} \Omega(\theta) P_{r w}(\theta) d \sigma(\theta)\right| d \sigma(w)<\infty .
$$

Definition 2.1 $\left(H^{1}\left(S^{n-1}\right)\right.$ atoms $)$ A function $b(\cdot)$ on $S^{n-1}$ is a regular atom if there exist $\varepsilon \in \mathrm{S}^{n-1}$ and $\varrho \in(0,2]$ such that

(i) $\operatorname{supp}(b) \subset S^{n-1} \cap B(\varepsilon, \varrho)$, where $B(\varepsilon, \varrho)=\left\{y \in \mathbb{R}^{n}:|y-\varepsilon|<\varrho\right\}$;

(ii) $\|b\|_{L^{2}\left(S^{n-1}\right)} \leq \varrho^{(1-n) / 2}$;

(iii) $\int_{S^{n-1}} b(y) d \sigma(y)=0$.

The following atomic decomposition of Hardy space can be obtained by the idea in $[7,8]$.

Lemma 2.1 ([8]) If $\Omega \in H^{1}\left(\mathrm{~S}^{n-1}\right)$ satisfies the cancelation condition (1.1), then there exist a sequence of complex numbers $\left\{c_{j}\right\}$ and a sequence of $(1, \infty)$ atoms $\left\{\Omega_{j}\right\}$ such that $\Omega=$ $\sum_{j} c_{j} \Omega_{j}$ and $\|\Omega\|_{H^{1}\left(S^{n-1}\right)} \approx \sum_{j}\left|c_{j}\right|$.

In our proofs of the main results we shall encounter oscillatory integrals with generalized polynomials as their phase functions. Thus the following lemma of van der Corput type is needed.

Lemma 2.2 ([6]) Let $l \in \mathbb{N} \backslash\{0\}, \mu_{1}, \ldots, \mu_{l} \in \mathbb{R}$, and $d_{1}, \ldots, d_{l}$ be distinct nonzero real numbers. Let $\psi \in \mathcal{C}^{1}([1 / 2,1])$. Then

$$
\left|\int_{\delta}^{\tau} \exp \left(i\left(\mu_{1} t^{d_{1}}+\cdots+\mu_{l} t^{d_{l}}\right)\right) \psi(t) d t\right| \leq C\left|\mu_{1}\right|^{-1 / l}\left(|\psi(\tau)|+\int_{\delta}^{\tau}\left|\psi^{\prime}(t)\right| d t\right)
$$

holds for $1 / 2 \leq \delta<\tau \leq 1$. Here, $C>0$ is independent of $\left\{\mu_{j}\right\}_{j=1}^{l}$.

Applying Lemma 2.2 and the arguments similar to those used in deriving [6, Lemma 2.5], we can get the following lemma.

Lemma 2.3 Let $l \in \mathbb{N} \backslash\{0\}, s \in \mathbb{N} \backslash\{0\}$, and $h_{1}, \ldots, h_{l}$ be distinct nonzero real numbers and

$$
Q(t, u)=t^{h_{1}} \sum_{|\alpha| \leq s} a_{\alpha} u^{\alpha}+\sum_{j=2}^{l} t^{h_{j}} w_{j}(u)
$$

where $t \in \mathbb{R}, u=\left(u_{1}, \ldots, u_{n-1}\right) \in \mathbb{R}^{n-1}, \alpha \in \mathbb{N}^{n-1}, a_{\alpha} \in \mathbb{R}$, and $w_{j}(\cdot)$ are real-valued. Let $r>0$ and $b(\cdot)$ be a measurable function on $[-r, r]^{n-1}$ that satisfies $\|b\|_{L^{2}\left([-r, r]^{n-1}\right)} \leq r^{-(n-1) / 2}$. Then there exists a positive constant $C$ independent of $\left\{a_{\alpha}\right\},\left\{w_{j}(\cdot)\right\}, r$ such that

$$
\int_{1 / 2}^{1}\left|\int_{[-r, r]^{n-1}} \exp (i Q(t, u)) b(u) d u\right| d t \leq C\left(r^{s} \sum_{|\alpha|=s}\left|a_{\alpha}\right|\right)^{-\gamma},
$$

where $\gamma=\frac{1}{2} \min \left\{1 / h_{1}, 1 / l, 1 /(s+1)\right\}$.

Proof Without loss of generality, we may assume that $\left|a_{(s, 0, \ldots, 0)}\right| \approx \sum_{|\alpha|=s}\left|a_{\alpha}\right|$. Let $\eta=$ $\left(u_{2}, \ldots, u_{n-1}\right)$ and $R(u)=\sum_{|\alpha| \leq s} a_{\alpha} u^{\alpha}$. By a change of variable and Hölder's inequality, we 
have

$$
\begin{aligned}
& \int_{1 / 2}^{1}\left|\int_{[-r, r]^{n-1}} \exp (i Q(t, u)) b(u) d u\right| d t \\
& =r^{n-1} \int_{1 / 2}^{1}\left|\int_{[-1,1]^{n-1}} \exp (i Q(t, r u)) b(r u) d u\right| d t \\
& \leq r^{n-1} \int_{[-1,1]^{n-2}}\left(\int_{1 / 2}^{1}\left|\int_{-1}^{1} \exp \left(i Q\left(t, r u_{1}, r \eta\right)\right) b\left(r u_{1}, r \eta\right) d u_{1}\right|^{2} d t\right)^{1 / 2} d \eta \\
& \leq r^{n-1} \int_{[-1,1]^{n-2}}\left(\int_{-1}^{1} \int_{-1}^{1}\left|\int_{1 / 2}^{1} \exp \left(i\left(Q\left(t, r u_{1}, r \eta\right)-Q\left(t, r v_{1}, r \eta\right)\right)\right) d t\right|\right. \\
& \left.\times\left|b\left(r u_{1}, r \eta\right) \overline{b\left(r v_{1}, r \eta\right)}\right| d u_{1} d v_{1}\right)^{1 / 2} d \eta \\
& \leq r^{n-1} \int_{[-1,1]^{n-2}}\left(\int_{-1}^{1} \int_{-1}^{1}\left|\int_{1 / 2}^{1} \exp \left(i\left(Q\left(t, r u_{1}, r \eta\right)-Q\left(t, r v_{1}, r \eta\right)\right)\right) d t\right|^{2} d u_{1} d v_{1}\right)^{1 / 4} \\
& \times\left(\int_{-1}^{1} \int_{-1}^{1}\left|b\left(r u_{1}, r \eta\right) \overline{b\left(r v_{1}, r \eta\right)}\right|^{2} d u_{1} d v_{1}\right)^{1 / 4} d \eta \text {. }
\end{aligned}
$$

Applying Lemma 2.2, we obtain

$$
\left|\int_{1 / 2}^{1} \exp \left(i\left(Q\left(t, r u_{1}, r \eta\right)-Q\left(t, r v_{1}, r \eta\right)\right)\right) d t\right| \leq C\left|R\left(r u_{1}, r \eta\right)-R\left(r v_{1}, r \eta\right)\right|^{-2 \gamma}
$$

where $\gamma=\frac{1}{2} \min \left\{1 / h_{1}, 1 / l, 1 /(s+1)\right\}$. Note that

$$
R\left(r u_{1}, r \eta\right)=a_{(s, 0, \ldots, 0)} r^{s} u_{1}^{s}+\text { lower powers in } u_{1}
$$

Then, by applying an inequality proved by Ricci and Stein (see [23, p. 182]), we get from (2.2) and (2.3) that

$$
\begin{aligned}
& \int_{-1}^{1}\left|\int_{1 / 2}^{1} \exp \left(i\left(Q\left(t, r u_{1}, r \eta\right)-Q\left(t, r v_{1}, r \eta\right)\right)\right) d t\right|^{2} d u_{1} \\
& \quad \leq \int_{-1}^{1}\left|R\left(r u_{1}, r \eta\right)-R\left(r v_{1}, r \eta\right)\right|^{-4 \gamma} d u_{1} \leq C\left(r^{s} \sum_{|\alpha|=s}\left|a_{\alpha}\right|\right)^{-4 \gamma},
\end{aligned}
$$

where $C>0$ is independent of $v_{1}, \eta, r$. On the other hand, by Hölder's inequality and a change of variable, we have

$$
\begin{aligned}
& \int_{[-1,1]^{n-2}}\left(\int_{-1}^{1} \int_{-1}^{1}\left|b\left(r u_{1}, r \eta\right) \overline{b\left(r v_{1}, r \eta\right)}\right|^{2} d u_{1} d v_{1}\right)^{1 / 4} d \eta \\
& \quad \leq \int_{[-1,1]^{n-2}}\left(\int_{-1}^{1}\left|b\left(r u_{1}, r \eta\right)\right|^{2} d u_{1}\right)^{1 / 2} d \eta \\
& \quad \leq C\left(\int_{[-1,1]^{n-1}}|b(r u)|^{2} d u\right)^{1 / 2} \leq C r^{-(n-1) / 2}\|b\|_{L^{2}\left([-r,]^{n-1}\right)} \leq C r^{-(n-1)}
\end{aligned}
$$

where $C>0$ is independent of $r$. (2.5) together with (2.2) and (2.4) yields (2.1). 
Lemma 2.4 ([6]) For $j \in\{1,2\}$, let $U_{j}$ be a domain in $\mathbb{R}^{n_{j}}$ and $K_{j}$ be a compact subset of $U_{j}$. Let $h(\cdot, \cdot)$ be a real-analytic function on $U_{1} \times U_{2}$ such that $h(\cdot, z)$ is a nonzero function for every $z \in U_{2}$. Then there exists a positive number $\delta=\delta\left(h, K_{1}, K_{2}\right)$ such that

$$
\sup _{z \in K_{2}} \int_{K_{1}}|h(w, z)|^{-\delta} d w<\infty .
$$

\section{Proof of Theorem 1.1}

In order to prove Theorem 1.1, we need the following lemma, which is the main tool of proving Theorem 1.1.

Lemma 3.1 ([19]) Let $A>0, \Lambda \in \mathbb{N} \backslash\{0\}$ and $\left\{\sigma_{k, s}: 0 \leq s \leq \Lambda\right.$ and $\left.k \in \mathbb{Z}\right\}$ be a family of uniformly bounded Borel measures on $\mathbb{R}^{n}$ with $\sigma_{k, 0}(\xi)=0$ for every $k \in \mathbb{Z}$ and $\xi \in \mathbb{R}^{n}$. For $1 \leq s \leq \Lambda$, let $v_{s}, \eta_{s}>1, \delta_{s}, \beta_{s}>0,\left\{a_{k, s}\right\}$ be a sequence of real positive numbers, $\ell_{s} \in \mathbb{N} \backslash\{0\}$ and $L_{s}: \mathbb{R}^{n} \rightarrow \mathbb{R}^{\ell_{s}}$ be a linear transformation. Suppose that

(i) $\max \left\{\left|\widehat{\sigma_{k, s}}(\xi)\right|,|| \widehat{\sigma_{k, s}}|(\xi)|\right\} \leq C A \min \left\{1,\left|a_{k, s}^{v_{s}} L_{s}(\xi)\right|^{-\delta_{s} / v_{s}}\right\}$;

(ii) $\max \left\{\left|\widehat{\sigma_{k, s}}(\xi)-\widehat{\sigma_{k, s-1}}(\xi)\right|,|| \widehat{\sigma_{k, s}}\left|(\xi)-\widehat{\sigma_{k, s-1} \mid}(\xi)\right|\right\} \leq C A\left|a_{k, s}^{v_{s}} L_{s}(\xi)\right|^{\beta_{s} / v_{s}}$;

(iii) for every $q>1$, there exists $C_{q}>0$ independent of $\left\{L_{s}\right\}_{s=1}^{\Lambda}$ such that

$$
\left\|\sup _{k \in \mathbb{Z}}|| \sigma_{k, 0}|* f|\right\|_{L^{q\left(\mathbb{R}^{n}\right)}} \leq C_{q} A\|f\|_{L^{q\left(\mathbb{R}^{n}\right)}}
$$

(iv) for any $1 \leq s \leq \Lambda,\left\{a_{k, s}\right\}$ satisfies one of the following conditions:
(a) $\inf _{k \in \mathbb{Z}} \frac{a_{k+1, s}}{a_{k, s}} \geq \eta_{s}$,
(b) $\quad \inf _{k \in \mathbb{Z}} \frac{a_{k, s}}{a_{k+1, s}} \geq \eta_{s}$.

Then, for any $1<p<\infty$, there exists $C_{p}>0$ such that

$$
\max \left\{\left\|\sup _{k \in \mathbb{Z}}|| \sigma_{k, \Lambda}|* f|\right\|_{L^{p}\left(\mathbb{R}^{n}\right)},\left\|\sup _{k \in \mathbb{Z}}\left|\sum_{j=k}^{\infty} \sigma_{j, \Lambda} * f\right|\right\|_{L^{p}\left(\mathbb{R}^{n)}\right.}\right\} \leq C_{p} A\|f\|_{L^{p}\left(\mathbb{R}^{n)} .\right.}
$$

Here, the constant $C_{p}$ is independent of $\left\{L_{s}\right\}_{\lambda=1}^{\Lambda}$ and $\left\{v_{s}\right\}_{s=1}^{\Lambda}$, but depends on $\Lambda, p,\left\{\delta_{s}\right\}_{s=1}^{\Lambda},\left\{\beta_{s}\right\}_{s=1}^{\Lambda}$, and $\left\{\eta_{s}\right\}_{s=1}^{\Lambda}$.

We now proceed with the proof of Theorem 1.1. For notational convenience, we denote by $\mathcal{V}_{n-1}$ the set of polynomials in $n-1$ variables with real coefficients and set $[x]:=\max \{k \in$ $\mathbb{Z}: k \leq x\}$ for any $x \in \mathbb{R}$. For $s \in \mathbb{N}$, let $\mathcal{V}_{n-1, s}$ denote the subset of $\mathcal{V}_{n-1}$ which contains homogeneous polynomials of degree $s$.

Proof of Theorem 1.1 By Lemma 2.1 and Minkowski's inequality, it suffices to show that

$$
\max \left\{\left\|T_{\Omega, \Phi}^{*} f\right\|_{L^{p}\left(\mathbb{R}^{d}\right)},\left\|\mathrm{M}_{\Omega, \Phi}\right\|_{L^{p}\left(\mathbb{R}^{d}\right)}\right\} \leq C_{p}\|f\|_{L^{p}\left(\mathbb{R}^{d}\right)},
$$

where $\Omega$ is an $H^{1}$ atom on $\mathrm{S}^{n-1}$ satisfying conditions (i)-(iii) of Definition 2.1.

In what follows, we assume that $\Omega$ is an $H^{1}$ atom on $S^{n-1}$ satisfying conditions (i)-(iii) of Definition 2.1 with $\varrho \in(0,2]$. Without loss of generality, we may assume the following:

(i) $\lambda$ is the number of distinct $m_{j}$; 
(ii) $\Phi=\left(\Phi_{1}, \ldots, \Phi_{d}\right)=\left(\Phi^{1}, \ldots, \Phi^{\lambda}\right)$, where $\Phi^{s}=\left(\Phi_{s, 1}, \ldots, \Phi_{s, a_{s}}\right)$ with $\Phi_{s, j}(t y)=t^{m_{r_{s}}} \Phi_{s, j}(y)$ for any $1 \leq s \leq \lambda$ and $1 \leq j \leq a_{s}$;

(iii) $\left\{\Phi_{s, 1}, \ldots, \Phi_{s, o_{s}}\right\}$ forms a basis for $\operatorname{span}\left\{\Phi_{s, 1}, \ldots, \Phi_{s, a_{s}}\right\}$ for any $1 \leq s \leq \lambda$.

From assumption (ii) we see that $\sum_{s=1}^{\lambda} a_{s}=d$ and $\left\{r_{1}, \ldots, r_{\lambda}\right\} \subset\{1, \ldots, d\}$. We get from assumption (iii) that, for any $1 \leq s \leq \lambda$ and $1 \leq j \leq a_{s}$, there exists a sequence of numbers $\left\{b_{s, j, k}\right\}_{k=1}^{o_{s}}$ such that

$$
\Phi_{s, j}(y)=b_{s, j, 1} \Phi_{s, 1}(y)+\cdots+b_{s, j, o_{s}} \Phi_{s, o_{s}}(y)
$$

Let $\xi=\left(\xi_{1}, \ldots, \xi_{d}\right)=\left(\xi^{1}, \ldots, \xi^{\lambda}\right)$ with $\xi^{s}=\left(\xi_{s, 1}, \ldots, \xi_{s, a_{s}}\right)$ for $1 \leq s \leq \lambda$. For any $1 \leq s \leq \lambda$, let $\tilde{\Phi}^{s}=\left(\Phi_{s, 1}, \ldots, \Phi_{s, o_{s}}\right)$ and $\tilde{\xi}^{s}=\left(\xi_{s, 1}, \ldots, \xi_{s, o_{s}}\right)$. We define two sequences of linear transformations $\left\{H_{s, i}\right\}_{i=1}^{a_{s}}: \mathbb{R}^{o_{s}} \rightarrow \mathbb{R}$ and $\left\{R_{s, j}\right\}_{j=1}^{o_{s}}: \mathbb{R}^{a_{s}} \rightarrow \mathbb{R}$ as follows:

$$
\begin{array}{ll}
H_{s, i}\left(\tilde{\xi}^{s}\right)=b_{s, i, 1} \xi_{s, 1}+\cdots+b_{s, i, o_{s}} \xi_{s, o_{s}}, & 1 \leq i \leq a_{s} \\
R_{s, j}\left(\xi^{s}\right)=b_{s, 1, j} \xi_{s, 1}+\cdots+b_{s, a_{s}, j} \xi_{s, a_{s}}, & 1 \leq j \leq o_{s} .
\end{array}
$$

Define two sequences of linear transformations $\left\{H_{s}\right\}_{s=1}^{\lambda}$ and $\left\{R_{s}\right\}_{s=1}^{\lambda}$ by

$$
H_{s}\left(\tilde{\xi}^{s}\right)=\left(H_{s, 1}\left(\tilde{\xi}^{s}\right), \ldots, H_{s, a_{s}}\left(\tilde{\xi}^{s}\right)\right), \quad R_{s}\left(\xi^{s}\right)=\left(R_{s, 1}\left(\xi^{s}\right), \ldots, R_{s, o_{s}}\left(\xi^{s}\right)\right)
$$

It is not difficult to see that

$$
\xi^{s} \cdot \Phi^{s}=\xi^{s} \cdot H_{s}\left(\tilde{\Phi}^{s}\right)=R_{s}\left(\xi^{s}\right) \cdot \tilde{\Phi}^{s}, \quad 1 \leq s \leq \lambda
$$

For any $1 \leq s \leq \lambda$ and $z \in S^{o_{s}-1}$, we have that $z \cdot \tilde{\Phi}^{i}(\cdot)$ is a nonzero real-analytic function since $\left\{\Phi_{s, 1}, \ldots, \Phi_{s, o_{s}}\right\}$ is linearly independent. Invoking Lemma 2.4, there exists $\delta_{s}>0$ such that

$$
\sup _{z \in \mathrm{S}^{o_{s}-1}} \int_{\mathrm{S}^{n-1}}\left|z \cdot \tilde{\Phi}^{s}(y)\right|^{-\delta_{s}} d \sigma(y)<\infty
$$

Let $\epsilon_{s}=\min \left\{1 / s, \delta_{s} / 2\right\}$. It follows from [9, (5.30)], for any $1 \leq s \leq \lambda$, that there exist an orthogonal $n \times n$ matrix $U$ such that $\varepsilon U=\mathbf{e}=(0, \ldots, 0,1) \in S^{n-1}$ and a polynomial $P_{s, j} \in$ $\mathcal{V}_{n-1}$ such that $\operatorname{deg}\left(P_{s, j}\right) \leq\left[\frac{n-1}{\epsilon_{s}}\right]$ and

$$
\left|\Phi_{s, j}\left(y U^{-1}\right)-P_{s, j}(\tilde{y})\right| \leq C \varrho^{(n-1) / \epsilon_{s}}
$$

for every $y \in B(\mathbf{e}, \varrho) \cap S^{n-1}$ and $1 \leq j \leq a_{s}$, where $\tilde{y}=\left(y_{1} /|y|, \ldots, y_{n-1} /|y|\right)$. For any $1 \leq s \leq \lambda$, let $\mathcal{P}_{s}=\left(P_{s, 1}, \ldots, P_{s, a_{s}}\right)$ and $\operatorname{deg}\left(\mathcal{P}_{s}\right)=\max _{1 \leq j \leq a_{s}} \operatorname{deg}\left(P_{s, j}\right)$. Then there are integers $0 \leq \Lambda_{s, 1}<$ $\cdots<\Lambda_{s, M_{s}} \leq \operatorname{deg}\left(\mathcal{P}_{s}\right)$ and $Q_{s, j, \Lambda_{s, l}} \in \mathcal{V}_{n-1, \Lambda_{s, l}}$ for $1 \leq j \leq a_{s}$ and $1 \leq l \leq M_{s}$ such that

$$
\mathcal{P}_{s}=\sum_{l=1}^{M_{s}} \mathcal{Q}_{s, \Lambda_{s, l}}
$$


where $\mathcal{Q}_{s, \Lambda_{s, l}}=\left(Q_{s, 1, \Lambda_{s, l}}, \ldots, Q_{s, a_{s}, \Lambda_{s, l}}\right)$ and $\mathcal{Q}_{s, \Lambda_{s, l}} \neq(0, \ldots, 0)$ for any $1 \leq l \leq M_{s}$. For any $1 \leq$ $l \leq M_{s}$, let $\tilde{\mathcal{Q}}_{s, \Lambda_{s, l}}=\left(Q_{s, 1, \Lambda_{s, l}}, \ldots, Q_{s, o_{s}, \Lambda_{s, l}}\right)$ and

$$
\tilde{\mathcal{P}}_{s}=\sum_{l=1}^{M_{s}} \tilde{\mathcal{Q}}_{s, \Lambda_{s, l}}
$$

It follows from (3.4) that

$$
\left|\tilde{\Phi}^{s}(y)-\tilde{\mathcal{P}}_{s}(\widetilde{y U})\right| \leq C \varrho^{(n-1) / \epsilon_{s}}
$$

for every $y \in B(\varepsilon, \varrho) \cap S^{n-1}$ and $1 \leq s \leq \lambda$. For $1 \leq s \leq \lambda, 1 \leq l \leq M_{s}$, and $1 \leq j \leq a_{s}$, we set

$$
Q_{s, j, \Lambda_{s, l}}(y)=\sum_{|\beta|=\Lambda_{s, l}} b_{s, j, l, \beta} y^{\beta}
$$

Let $\varpi(u)=\sum_{s=1}^{u}\left(M_{s}+1\right)$ for $1 \leq u \leq \lambda, \varpi(0)=0$, and define the mappings $\Theta_{0}, \ldots, \Theta_{\varpi(\lambda)}$ by

$$
\Theta_{0}(y)=(0, \ldots, 0), \quad \Theta_{\varpi(\lambda)}(y)=\Phi(y)
$$

and

$$
\begin{aligned}
& \Theta_{\varpi(u)+\theta}(y) \\
& \quad=\left(\Phi^{1}(y), \ldots, \Phi^{u}(y),|y|^{m_{r_{u+1}}} H_{u+1}\left(\sum_{l=1}^{\theta} \tilde{\mathcal{Q}}_{u+1, \Lambda_{u+1, l}}\left(|y|^{-1} \widetilde{y U}\right)\right), 0, \ldots, 0\right)
\end{aligned}
$$

for $0 \leq u \leq \lambda-1,0 \leq \theta<\varpi(u+1)-\varpi(u)$. In particular,

$$
\Theta_{\varpi(u)-1}(y)=\left(\Phi^{1}(y), \ldots, \Phi^{u-1}(y),|y|^{m_{r_{u}}} H_{u}\left(\tilde{\mathcal{P}}_{u}\left(|y|^{-1} \widetilde{y U}\right)\right), 0, \ldots, 0\right), \quad 1 \leq u \leq \lambda .
$$

For $0 \leq s \leq \varpi(\lambda)$, define two families of measures $\left\{\sigma_{k, s}\right\}$ and $\left\{\left|\sigma_{k, s}\right|\right\}$ by

$$
\int_{\mathbb{R}^{d}} f(x) d \sigma_{k, s}(x)=\int_{2^{k-1}<|y| \leq 2^{k}} f\left(\Theta_{s}(y)\right) \frac{\Omega(y /|y|)}{|y|^{n}} d y .
$$

The measure $\left|\sigma_{k, s}\right|$ is defined in the same way as $\sigma_{k, s}$, but with $\Omega$ replaced by $|\Omega|$. By (1.1) and (3.7) we have

$$
\sigma_{k, 0}(\xi)=0 \quad \text { for } k \in \mathbb{Z}
$$

It is easy to check that

$$
\max \left\{\left|\widehat{\sigma_{k, S}}(\xi)\right|,\left|\widehat{\sigma_{k, s}}\right|(\xi) \mid\right\} \leq C\|\Omega\|_{L^{1}\left(S^{n-1}\right)} \text {. }
$$

For any $1 \leq s \leq \varpi(\lambda)$, by a change of variable, we have

$$
\widehat{\sigma_{k, S}}(\xi)=\int_{2^{k-1}}^{2^{k}} \int_{S^{n-1}} \Omega\left(y^{\prime}\right) \exp \left(-2 \pi i \xi \cdot \Theta_{s}\left(t y^{\prime}\right)\right) d \sigma\left(y^{\prime}\right) \frac{d t}{t}
$$




$$
=\int_{1 / 2}^{1} \int_{S^{n-1}} \Omega\left(y^{\prime}\right) \exp \left(-2 \pi i \xi \cdot \Theta_{s}\left(2^{k} t y^{\prime}\right)\right) d \sigma\left(y^{\prime}\right) \frac{d t}{t} .
$$

Note that $2 \epsilon_{u} \leq \delta_{u}$. By (3.3), (3.12), Hölder's inequality and invoking Lemma 2.2, one finds that

$$
\begin{aligned}
\left|\widehat{\sigma_{k, \varpi}(u)}(\xi)\right| & \leq \int_{S^{n-1}}\left|\Omega\left(y^{\prime}\right)\right|\left|\int_{1 / 2}^{1} \exp \left(-2 \pi i \xi \cdot \Theta_{\varpi(u)}\left(2^{k} t y^{\prime}\right)\right) \frac{d t}{t}\right| d \sigma\left(y^{\prime}\right) \\
& \leq \int_{S^{n-1}}\left|\Omega\left(y^{\prime}\right)\right|\left|\int_{1 / 2}^{1} \exp \left(-2 \pi i \sum_{j=1}^{u} R_{j}\left(\xi^{j}\right) \cdot \tilde{\Phi}^{j}\left(y^{\prime}\right)\left(2^{k} t\right)^{m_{r_{j}}}\right) \frac{d t}{t}\right| d \sigma\left(y^{\prime}\right) \\
& \leq C\left|2^{k m_{r_{u}}} R_{u}\left(\xi^{u}\right)\right|^{-\epsilon_{u}} \int_{S^{n-1}}\left|\Omega\left(y^{\prime}\right)\right|\left|\left(R_{u}\left(\xi^{u}\right)\right)^{\prime} \cdot \tilde{\Phi}^{u}\left(y^{\prime}\right)\right|^{-\epsilon_{u}} d \sigma\left(y^{\prime}\right) \\
& \leq C\left|2^{k m_{r_{u}}} R_{u}\left(\xi^{u}\right)\right|^{-\epsilon_{u}}\|\Omega\|_{L^{2}\left(S^{n-1}\right)}\left(\int_{S^{n-1}}\left|\left(R_{u}\left(\xi^{u}\right)\right)^{\prime} \cdot \tilde{\Phi}^{u}\left(y^{\prime}\right)\right|^{-2 \epsilon_{u}} d \sigma\left(y^{\prime}\right)\right)^{1 / 2} \\
& \leq C\left|2^{k m_{r_{u}}} R_{u}\left(\xi^{u}\right)\right|^{-\epsilon_{u}}\|\Omega\|_{L^{2}\left(S^{n-1}\right)}\left(\int_{S^{n-1}}\left|\left(R_{u}\left(\xi^{u}\right)\right)^{\prime} \cdot \tilde{\Phi}^{u}\left(y^{\prime}\right)\right|^{-\delta_{u}} d \sigma\left(y^{\prime}\right)\right)^{1 / 2} \\
& \leq C\left|2^{k m_{r_{u}}} \varrho^{-(n-1) /\left(2 \epsilon_{u}\right)} R_{u}\left(\xi^{u}\right)\right|^{-\epsilon_{u}}
\end{aligned}
$$

for any $1 \leq u \leq \lambda$, where $\left(R_{u}\left(\xi^{u}\right)\right)^{\prime}=\frac{R_{u}\left(\xi^{u}\right)}{\left|R_{u}\left(\xi^{u}\right)\right|}$. Similarly, we can get

$$
\left.\left|\widehat{\sigma_{k, \varpi(u)}}\right|(\xi)|\leq C| 2^{k m_{r_{u}}} \varrho^{-(n-1) /\left(2 \epsilon_{u}\right)} R_{u}\left(\xi^{u}\right)\right|^{-\epsilon_{u}}
$$

for $1 \leq u \leq \lambda$.

For $0 \leq u \leq \lambda-1$ and $0<\theta<\varpi(u+1)-\varpi(u)$, we get from (3.8) and (3.12) that

$$
\begin{aligned}
\left|\widehat{\sigma_{k, \varpi(u)+\theta}}(\xi)\right|= & \mid \int_{1 / 2}^{1} \int_{S^{n-1}} \Omega\left(y^{\prime}\right) \exp \left(-2 \pi i\left(\sum_{\ell=1}^{u} \xi^{\ell} \cdot \Phi^{\ell}\left(y U^{-1}\right)\left(2^{k} t\right)^{m_{r_{\ell}}}\right.\right. \\
& \left.+\xi^{u+1} \cdot H_{u+1}\left(\sum_{l=1}^{\theta} \tilde{\mathcal{Q}}_{u+1, \Lambda_{u+1, l}}(\tilde{y})\left(2^{k} t\right)^{m_{r_{u+1}}}\right)\right) \frac{d t}{t} \mid
\end{aligned}
$$

From (3.2) and (3.6) we have

$$
\begin{aligned}
\xi^{u+1} \cdot H_{u+1}\left(\sum_{l=1}^{\theta} \tilde{\mathcal{Q}}_{u+1, \Lambda_{u+1, l}}(\tilde{y})\right) & =R_{u+1}\left(\xi^{u+1}\right) \cdot\left(\sum_{l=1}^{\theta} \tilde{\mathcal{Q}}_{u+1, \Lambda_{u+1, l}}(\tilde{y})\right) \\
& =\sum_{j=1}^{o_{u+1}} R_{u+1, j}\left(\xi^{u+1}\right) \cdot\left(\sum_{l=1}^{\theta} Q_{u+1, j, \Lambda_{u+1, l}}(\tilde{y})\right) \\
& =\sum_{l=1}^{\theta} \sum_{|\beta|=\Lambda_{u+1, l}}\left(\sum_{j=1}^{o_{u+1}} b_{u+1, j, l, \beta} R_{u+1, j}\left(\xi^{u+1}\right)\right)(\tilde{y})^{\beta} .
\end{aligned}
$$

Invoking Lemma 2.3, there exists a positive constant $\gamma_{u, \theta}$ such that, for $0 \leq u \leq \lambda-1$ and $0<\theta<\varpi(u+1)-\varpi(u)$,

$$
\left|\widehat{\sigma_{k, \varpi(u)+\theta}}(\xi)\right| \leq C\left|2^{k m_{r_{u+1}}} \varrho^{\Lambda_{u+1, \theta}} L^{\left(\Lambda_{u+1, \theta}\right)}(\xi)\right|^{-\gamma_{u, \theta}},
$$


where

$$
L^{\left(\Lambda_{u+1, \theta}\right)}(\xi)=\left(\sum_{j=1}^{o_{u+1}} b_{u+1, j, \theta, \beta} R_{u+1, j}\left(\xi^{u+1}\right)\right)_{|\beta|=\Lambda_{u+1, \theta}} .
$$

Similarly, we get that, for $0 \leq u \leq \lambda-1$ and $0<\theta<\varpi(u+1)-\varpi(u)$,

$$
|| \widehat{\sigma_{k, \varpi(u)+\theta}}|(\xi)| \leq C\left|2^{k m_{r_{u+1}}} \varrho^{\Lambda_{u+1, \theta}} L^{\left(\Lambda_{u+1, \theta}\right)}(\xi)\right|^{-\gamma_{u, \theta}} .
$$

On the other hand, by a change of variable, we get from (3.2), (3.5), and (3.8) that

$$
\begin{aligned}
& \max \left\{\left|\widehat{\sigma_{k, \varpi(u)}}(\xi)-\widehat{\sigma_{k, \varpi(u)-1}}(\xi)\right|,\left|\widehat{\sigma_{k, \varpi(u)}}\right|(\xi)-\left|\widehat{\sigma_{k, \varpi(u)-1}}\right|(\xi) \mid\right\} \\
& =\int_{2^{k-1}<|y| \leq 2^{k}}\left|\exp \left(-2 \pi i \xi \cdot \Theta_{\varpi(u)}(y)\right)-\exp \left(-2 \pi i \xi \cdot \Theta_{\varpi(u)-1}(y)\right)\right| \frac{|\Omega(y /|y|)|}{|y|^{n}} d y \\
& \leq C 2^{k m_{r_{u}}} \int_{S^{n-1}}|\Omega(y)|\left|\xi^{u} \cdot \Phi^{u}(y)-\xi^{u} \cdot H_{u}\left(\mathcal{P}_{u}(\widetilde{y U})\right)\right| d \sigma(y) \\
& \leq C 2^{k m_{r_{u}}} \int_{S^{n-1}}|\Omega(y)|\left|R_{u}\left(\xi^{u}\right) \cdot\left(\tilde{\Phi}^{u}(y)-\tilde{\mathcal{P}}_{u}(\widetilde{y U})\right)\right| d \sigma(y) \\
& \leq C\|\Omega\|_{L^{1}\left(S^{n-1}\right)} 2^{k m_{r_{u}}}\left|\varrho^{(n-1) / \epsilon_{u}} R_{u}\left(\xi^{u}\right)\right|
\end{aligned}
$$

for $1 \leq u \leq \lambda$. For $1 \leq u \leq \lambda-1$ and $1 \leq \theta<\varpi(u)-\varpi(u-1)$, we have

$$
\begin{aligned}
& \max \left\{\left|\widehat{\sigma_{k, \varpi(u)+\theta}}(\xi)-\sigma_{k, \sigma(u)+\theta-1}(\xi)\right|,|| \widehat{\sigma_{k, \varpi(u)+\theta}}|(\xi)-| \widehat{\sigma_{k, \varpi(u)+\theta}-1}|(\xi)|\right\} \\
& \quad=\int_{2^{k-1}<|y| \leq 2^{k}}\left|\exp \left(-2 \pi i \xi \cdot \Theta_{\varpi(u)+\theta}(y)\right)-\exp \left(-2 \pi i \xi \cdot \Theta_{\varpi(u)+\theta-1}(y)\right)\right| \frac{|\Omega(y /|y|)|}{|y|^{n}} d y \\
& \quad \leq C \int_{2^{k-1}<|y| \leq 2^{k}}\left|2^{k m_{r_{u+1}}} \xi^{u+1} \cdot H_{u+1}\left(\tilde{\mathcal{Q}}_{u+1, \Lambda_{u+1, \theta}}\left(|y|^{-1} \widetilde{y U}\right)\right)\right| \frac{|\Omega(y /|y|)|}{|y|^{n}} d y \\
& \quad \leq C\|\Omega\|_{L^{1}\left(S^{n-1}\right)} 2^{k m_{r_{u+1}}}\left|\varrho^{\Lambda_{u+1, \theta}} L^{\left(\Lambda_{u+1, \theta}\right)}(\xi)\right| .
\end{aligned}
$$

Define the sequence of linear transformations $\left\{L_{s}\right\}_{s=1}^{\varpi(\lambda)}$ by

$$
L_{s}(\xi):= \begin{cases}\varrho^{\Lambda_{u+1, \theta}} L^{\left(\Lambda_{u+1, \theta}\right)}(\xi), & s=\varpi(u)+\theta, 0 \leq u \leq \lambda-1,0<\theta<\varpi(u+1)-\varpi(u), \\ \varrho^{(n-1) /\left(2 \epsilon_{u}\right)} R_{u}\left(\xi^{u}\right), & s=\varpi(u), 1 \leq u \leq \lambda .\end{cases}
$$

Also, we define $N_{1}, \ldots, N_{\varpi(\lambda)}$ and $\eta_{1}, \ldots, \eta_{\varpi(\lambda)}$ by

$$
\begin{aligned}
& N_{s}:= \begin{cases}\gamma_{u, \theta}, & s=\varpi(u)+\theta, 0 \leq u \leq \lambda-1,0<\theta<\varpi(u+1)-\varpi(u), \\
\epsilon_{u}, & s=\varpi(u), 1 \leq u \leq \lambda ;\end{cases} \\
& \eta_{s}:= \begin{cases}m_{r_{u+1}}, & s=\varpi(u)+\theta, 0 \leq u \leq \lambda-1,0<\theta<\varpi(u+1)-\varpi(u), \\
m_{r_{u}}, & s=\varpi(u), 1 \leq u \leq \lambda .\end{cases}
\end{aligned}
$$

It follows from (3.11) and (3.13)-(3.18) that, for any $1 \leq s \leq \varpi(\lambda)$,

$$
\max \left\{\left|\widehat{\sigma_{k, s}}(\xi)-\widehat{\sigma_{k, s-1}}(\xi)\right|,\left|\widehat{\sigma_{k, s}}\right|(\xi)-\widehat{\left|\widehat{\sigma_{k, s-1}}\right|}(\xi) \mid\right\} \leq C\left|2^{k \eta_{s}} L_{s}(\xi)\right| ;
$$




$$
\max \left\{\left|\widehat{\sigma_{k, s}}(\xi)\right|,\left|\widehat{\sigma_{k, s}}\right|(\xi) \mid\right\} \leq C \min \left\{1,\left|2^{k \eta_{s}} L_{s}(\xi)\right|^{-N_{s}}\right\}
$$

On the other hand, we get from (3.7) that

$$
\sup _{k \in \mathbb{Z}}|| \sigma_{k, 0}|* f(x)| \leq C|f(x)|
$$

From (3.21) we have

$$
\left\|\sup _{k \in \mathbb{Z}}|| \sigma_{k, 0}|* f|\right\|_{L^{q}\left(\mathbb{R}^{d}\right)} \leq C\|f\|_{L^{q}\left(\mathbb{R}^{d}\right)}, \quad 1<q \leq \infty .
$$

Invoking Lemma 3.1, we get from (3.10), (3.11), (3.19), (3.20), and (3.22) that

$$
\begin{aligned}
& \left\|\sup _{k \in \mathbb{Z}}|| \sigma_{k, \varpi(\lambda)}|* f|\right\|_{L^{p}\left(\mathbb{R}^{d}\right)} \leq C\|f\|_{L^{p}\left(\mathbb{R}^{d}\right)}, \quad 1<p<\infty, \\
& \left\|\sup _{k \in \mathbb{Z}}\left|\sum_{j=k}^{\infty} \sigma_{j, \varpi(\lambda)} * f\right|\right\|_{L^{p}\left(\mathbb{R}^{d}\right)} \leq C\|f\|_{L^{p}\left(\mathbb{R}^{d}\right)}, \quad 1<p<\infty .
\end{aligned}
$$

For any $\epsilon>0$, there exists an integer $k$ such that $2^{k-1} \leq \epsilon<2^{k}$. It follows that

$$
T_{\Omega, \Phi}^{*} f \leq \sup _{k \in \mathbb{Z}}|| \sigma_{k, \varpi}(\lambda)|*| f||+\sup _{k \in \mathbb{Z}}\left|\sum_{j=k}^{\infty} \sigma_{j, \varpi(\lambda)} * f\right| .
$$

On the other hand, one can easily check that

$$
\mathrm{M}_{\Omega, \Phi} f(x) \leq C \sup _{k \in \mathbb{Z}}|| \sigma_{k, \varpi(\lambda)}|*| f|(x)| \quad \text { for } x \in \mathbb{R}^{d}
$$

Then (3.26) together with (3.23)-(3.25) yields (3.1) and completes the proof of Theorem 1.1 .

\section{Proof of Theorem 1.2}

This section is devoted to proving Theorem 1.2. Before presenting the proof of Theorem 1.2 , let us recall some useful lemmas. In what follows, we set $\mathfrak{R}_{d}=\left\{\xi \in \mathbb{R}^{d} ; 1 / 2<\right.$ $|\xi| \leq 1\}$.

Lemma 4.1 ([26]) Let $\alpha \in(0,1), p \in(1, \infty), q \in(1, \infty]$, and $r \in[1, \min \{p, q\})$. Then

$$
\|f\|_{\dot{F}_{\alpha}^{p, q}\left(\mathbb{R}^{d}\right)} \sim\left\|\left(\sum_{l \in \mathbb{Z}} 2^{l q \alpha}\left(\int_{\mathfrak{R}_{d}}\left|\Delta_{2^{-l} \zeta} f\right|^{r} d \zeta\right)^{q / r}\right)^{1 / q}\right\|_{L^{p}\left(\mathbb{R}^{d}\right)}
$$

Lemma 4.2 ([21]) Let $v \geq 1, \Lambda \in \mathbb{N} \backslash\{0\}$, and $\left\{\sigma_{k}^{s}: k \in \mathbb{Z}, 1 \leq s \leq \Lambda\right\}$ be a family of Borel measures on $\mathbb{R}^{d}$ with $\sigma_{k}^{0}=0$ for all $k \in \mathbb{Z}$. Let $\left|\sigma_{k}^{s}\right|$ be the total variation of $\sigma_{k}^{s}$. For $1 \leq s \leq \Lambda$, let $\beta_{s}, \gamma_{s}>0, M_{s} \in \mathbb{N} \backslash\{0\}$, and $L_{s}: \mathbb{R}^{d} \rightarrow \mathbb{R}^{M_{s}}$ be linear transformations. Suppose that there exist $C, A>0$ independent of $v$ such that, for $1 \leq s \leq \Lambda, k \in \mathbb{Z}$, and $\xi \in \mathbb{R}^{d}$, the following conditions are satisfied:

(a) $\max \left\{\left|\widehat{\sigma_{k}^{s}}(\xi)-\widehat{\sigma_{k}^{s-1}}(\xi)\right|,\left|\widehat{\left|\sigma_{k}^{s}\right|}\right|(\xi)-\widehat{\mid \sigma_{k}^{s-1}}|(\xi)|\right\} \leq C A\left|2^{k v \gamma_{s}} L_{s}(\xi)\right|^{1 / v}$

(b) $\max \left\{\left|\widehat{\sigma_{k}^{s}}(\xi)\right|,\left|\widehat{\left|\sigma_{k}^{s}\right|}(\xi)\right|\right\} \leq C A \min \left\{1,\left|2^{k v \gamma_{s}} L_{s}(\xi)\right|^{-\beta_{s} / v}\right\}$; 
(c) There exists $\vartheta \in \mathbb{R}^{d}$ such that $\sup _{k \in \mathbb{Z}}|| \sigma_{k}^{0}|* f(x)| \leq C A|f(x+\vartheta)|$ for any $k \in \mathbb{Z}$;

(i) Suppose that there exist $p_{0}, q_{0}>1$ satisfying $\left(p_{0}, q_{0}\right) \neq(2,2), 1<r_{0}<\min \left\{p_{0}, q_{0}\right\}$, and $2 \leq u<\infty$ such that the following condition (d) holds for $1 \leq s \leq \Lambda$ :

(d)

$$
\begin{aligned}
& \left\|\left(\sum_{l \in \mathbb{Z}}\left\|\left(\sum_{k \in \mathbb{Z}} \| \sigma_{k}^{s}\left|* g_{l, \zeta, k}\right|^{u}\right)^{1 / u}\right\|_{L^{r_{0}\left(\Re_{d}\right)}}^{q_{0}}\right)^{1 / q_{0}}\right\|_{L^{p_{0}\left(\mathbb{R}^{d}\right)}} \\
& \leq C A\left\|\left(\sum_{l \in \mathbb{Z}}\left\|\left(\sum_{k \in \mathbb{Z}}\left|g_{l, \zeta, k}\right|^{u}\right)^{1 / u}\right\|_{L^{r_{0}\left(\Re_{d}\right)}}^{q_{0}}\right)^{1 / q_{0}}\right\|_{L^{p_{0}\left(\mathbb{R}^{d}\right)}} .
\end{aligned}
$$

Let $P_{1} P_{2}$ be the line segment from $P_{1}$ to $P_{2}$ with $P_{1}=\left(\frac{1}{2}, \frac{1}{2}\right)$ and $P_{2}=\left(\frac{1}{p_{0}}, \frac{1}{q_{0}}\right)$. Then, for $1 \leq s \leq \Lambda, \alpha \in(0,1)$, and $\left(\frac{1}{p}, \frac{1}{q}\right) \in P_{1} P_{2} \backslash\left\{\left(\frac{1}{p_{0}}, \frac{1}{q_{0}}\right)\right\}$, there exists $C>0$ independent of $A$ and $v$ such that

$$
\left\|\left(\sum_{l \in \mathbb{Z}} 2^{l q \alpha}\left(\int_{\mathfrak{R}_{d}} \sup _{k \in \mathbb{Z}}|| \sigma_{k}^{s}|*| \Delta_{2^{-l} \zeta} f|| d \zeta\right)^{q}\right)^{1 / q}\right\|_{L^{p}\left(\mathbb{R}^{d}\right)} \leq C A\|f\|_{\dot{F}_{\alpha}^{p, q}\left(\mathbb{R}^{d}\right)} .
$$

(ii) Suppose also that the following condition (e) holds for $1 \leq s \leq \Lambda$ :

(e)

$$
\left\|\left(\sum_{j \in \mathbb{Z}}\left(\sum_{k \in \mathbb{Z}}\left|\sigma_{k}^{s} * g_{k, j}\right|^{2}\right)^{q_{0} / 2}\right)^{1 / q_{0}}\right\|_{L^{p_{0}\left(\mathbb{R}^{d}\right)}} \leq C A\left\|\left(\sum_{j \in \mathbb{Z}}\left(\sum_{k \in \mathbb{Z}}\left|g_{k, j}\right|^{2}\right)^{q_{0} / 2}\right)^{1 / q_{0}}\right\|_{L^{p_{0}\left(\mathbb{R}^{d}\right)}} .
$$

Then, for $\alpha \in(0,1)$ and $\left(\frac{1}{p}, \frac{1}{q}\right) \in P_{1} P_{2} \backslash\left\{\left(\frac{1}{p_{0}}, \frac{1}{q_{0}}\right),\left(\frac{1}{2}, \frac{1}{2}\right)\right\}$, there exists a constant $C>0$ independent of $A$ and $v$ such that

$$
\left\|\left(\sum_{l \in \mathbb{Z}} 2^{l q \alpha}\left(\int_{\mathfrak{R}_{d}} \sup _{k \in \mathbb{Z}}\left|\sum_{j=k}^{\infty} \sigma_{j}^{\Lambda} * \Delta_{2^{-l_{\zeta}}} f\right| d \zeta\right)^{q}\right)^{1 / q}\right\|_{L^{p}\left(\mathbb{R}^{d}\right)} \leq C A\|f\|_{\dot{F}_{\alpha}^{p, q}\left(\mathbb{R}^{d}\right)} .
$$

We now give the proof of Theorem 1.2.

Proof of Theorem 1.2 We first prove the boundedness part. One can easily check that $T_{\Omega, \Phi}^{*}$ and $\mathrm{M}_{\Omega, \Phi}$ satisfy condition (1.7). This together with Lemma 4.1 yields that

$$
\begin{aligned}
\left\|T_{\Omega, \Phi}^{*} f\right\|_{F_{\alpha}^{p, q}\left(\mathbb{R}^{d}\right)} & \leq C\left\|\left(\sum_{l \in \mathbb{Z}} 2^{l q \alpha}\left(\int_{\mathfrak{R}_{d}}\left|\Delta_{2^{-l} l_{\zeta}}\left(T_{\Omega, \Phi}^{*} f\right)\right| d \zeta\right)^{q}\right)^{1 / q}\right\|_{L^{p}\left(\mathbb{R}^{d}\right)} \\
& \leq C\left\|\left(\sum_{l \in \mathbb{Z}} 2^{l q \alpha}\left(\int_{\mathfrak{\Re}_{d}}\left|T_{\Omega, \Phi}^{*}\left(\Delta_{2^{-} l_{\zeta}} f\right)\right| d \zeta\right)^{q}\right)^{1 / q}\right\|_{L^{p}\left(\mathbb{R}^{d}\right)}, \\
\left\|\mathrm{M}_{\Omega, \Phi} f\right\|_{\dot{F}_{\alpha}^{p, q}\left(\mathbb{R}^{d}\right)} & \leq C\left\|\left(\sum_{l \in \mathbb{Z}} 2^{l q \alpha}\left(\int_{\mathfrak{R}_{d}}\left|\mathrm{M}_{\Omega, \Gamma}\left(\Delta_{2^{-l} l_{\zeta}} f\right)\right| d \zeta\right)^{q}\right)^{1 / q}\right\|_{L^{p}\left(\mathbb{R}^{d}\right)}
\end{aligned}
$$

for $\alpha \in(0,1)$ and $1<p, q<\infty$. By Lemma 2.1 and Minkowski's inequality, it suffices to show that

$$
\left\|\left(\sum_{l \in \mathbb{Z}} 2^{l q \alpha}\left(\int_{\mathfrak{R}_{d}}\left|T_{\Omega, \Phi}^{*}\left(\Delta_{2^{-} l_{\zeta}} f\right)\right| d \zeta\right)^{q}\right)^{1 / q}\right\|_{L^{p}\left(\mathbb{R}^{d}\right)} \leq C\|f\|_{\dot{F}_{\alpha}^{p, q}\left(\mathbb{R}^{d}\right)},
$$




$$
\left\|\left(\sum_{l \in \mathbb{Z}} 2^{l q \alpha}\left(\int_{\mathfrak{R}_{d}}\left|\mathrm{M}_{\Omega, \Phi}\left(\Delta_{2^{-l} \zeta} f\right)\right| d \zeta\right)^{q}\right)^{1 / q}\right\|_{L^{p}\left(\mathbb{R}^{d}\right)} \leq C\|f\|_{\dot{F}_{\alpha}^{p, q}\left(\mathbb{R}^{d}\right)}
$$

where $\Omega$ is an $H^{1}$ atom on $S^{n-1}$ satisfying conditions (i)-(iii) of Definition 2.1. Without loss of generality, we may assume that $\Omega, \Phi$ are given as in the proof of Theorem 1.1. We also let $\left\{\sigma_{k, s}\right\}_{s=0}^{\sigma^{(\lambda)}}$ and $\left\{\mid \sigma_{k, s}\right\}_{s=1}^{\varpi(\lambda)}$ be defined as in the proof of Theorem 1.1. By [22, Lemmas 2.4-2.5], we obtain that, for any $1 \leq s \leq \varpi(\lambda)$ and $1<p, q, r<\infty$,

$$
\begin{aligned}
& \left\|\left(\sum_{j \in \mathbb{Z}}\left(\sum_{k \in \mathbb{Z}}\left|\sigma_{k, s} * g_{k, j}\right|^{2}\right)^{q / 2}\right)^{1 / q}\right\|_{L^{p}\left(\mathbb{R}^{d}\right)} \leq C\left\|\left(\sum_{j \in \mathbb{Z}}\left(\sum_{k \in \mathbb{Z}}\left|g_{k, j}\right|^{2}\right)^{q / 2}\right)^{1 / q}\right\|_{L^{p}\left(\mathbb{R}^{d}\right)} ; \\
& \left\|\left(\sum_{j \in \mathbb{Z}}\left\|\left(\sum_{k \in \mathbb{Z}}|| \sigma_{k, s}\left|* g_{j, \zeta, k}\right|^{2}\right)^{1 / 2}\right\|_{L^{r}\left(\Re_{d}\right)}^{q}\right)^{1 / q}\right\|_{L^{p}\left(\mathbb{R}^{d}\right)} \\
& \quad \leq C\left\|\left(\sum_{j \in \mathbb{Z}}\left\|\left(\sum_{k \in \mathbb{Z}}\left|g_{j, \zeta, k}\right|^{2}\right)^{1 / 2}\right\|_{L^{r}\left(\Re_{d}\right)}^{q}\right)^{1 / q}\right\|_{L^{p}\left(\mathbb{R}^{d}\right)} .
\end{aligned}
$$

By (3.10), (3.19)-(3.21), (4.5), (4.6) and applying Lemma 4.2, we have that, for $\alpha \in(0,1)$ and $1<p, q<\infty$,

$$
\begin{aligned}
& \left\|\left(\sum_{l \in \mathbb{Z}} 2^{l q \alpha}\left(\int_{\mathfrak{R}_{d}} \sup _{k \in \mathbb{Z}}|| \sigma_{k, \sigma(\lambda)}|*| \Delta_{2^{-} l_{\zeta}} f|| d \zeta\right)^{q}\right)^{1 / q}\right\|_{L^{p}\left(\mathbb{R}^{d}\right)} \leq C\|f\|_{\dot{F}_{\alpha}^{p, q}\left(\mathbb{R}^{d}\right)}, \\
& \left\|\left(\sum_{l \in \mathbb{Z}} 2^{l q \alpha}\left(\int_{\mathfrak{R}_{d}} \sup _{k \in \mathbb{Z}}\left|\sum_{j=k}^{\infty} \sigma_{j, \varpi(\lambda)} * \Delta_{2^{-} l_{\zeta}} f\right| d \zeta\right)^{q}\right)^{1 / q}\right\|_{L^{p}\left(\mathbb{R}^{d}\right)} \leq C\|f\|_{\dot{F}_{\alpha}^{p, q}\left(\mathbb{R}^{d}\right)} .
\end{aligned}
$$

Then (4.1) follows from (3.25), (4.7), and (4.8). Inequality (3.26) together with (4.7) yields (4.2). One can easily check that both the operators $T_{\Omega, \Phi}^{*}$ and $M_{\Omega, \Phi}$ satisfy conditions (1.7) and (1.8). This together with (1.4), (4.1), (4.2), Theorem 1.1, and the arguments similar to those used to derive the continuity part in [22, Theorem 1.1]. We omit the details.

\section{Acknowledgements}

The authors want to express their sincere thanks to the referee for his or her valuable remarks and suggestions, which made this paper more readable.

Funding

This work was supported partially by the NNSF of China (No. 11701333).

Availability of data and materials

Not applicable.

Competing interests

The authors declare that they have no competing interests.

Authors' contributions

The authors worked jointly in drafting and approving the final manuscript.

\section{Author details}

${ }^{1}$ College of Electronic and Information Engineering, Shandong University of Science and Technology, Qingdao, 266590, P.R. China. ${ }^{2}$ College of Mathematics and System Science, Shandong University of Science and Technology, Qingdao, 266590, P.R. China.

\section{Publisher's Note}

Springer Nature remains neutral with regard to jurisdictional claims in published maps and institutional affiliations. 


\section{References}

1. Al-Qassem, A., Cheng, L., Pan, Y.: Boundedness of rough integral operators on Triebel-Lizorkin spaces. Publ. Math. 56, 261-277 (2012)

2. Al-Salman, A., Pan, Y.: Singular integrals with rough kernels in $L \log L\left(\mathbf{S}^{n-1}\right)$. J. Lond. Math. Soc. 66, 153-174 (2002)

3. Calderón, A.P., Zygmund, A.: On singular integral. Am. J. Math. 78, 289-309 (1956)

4. Chen, J., Fan, D., Ying, Y.: Singular integral operators on function spaces. J. Math. Anal. Appl. 276, 691-708 (2002)

5. Chen, Y., Ding, Y., Liu, H.: Rough singular integrals supported on submanifolds. J. Math. Anal. Appl. 368, 677-691 (2010)

6. Cheng, L.C.: Singular integrals related to homogeneous mappings. Mich. Math. J. 47, 407-416 (2000)

7. Coifman, R., Weiss, G.: Extension of Hardy spaces and their use in analysis. Bull. Am. Math. Soc. 83, 569-645 (1977)

8. Connett, W.C.: Singular integral near L'. In: Harmonic Analysis in Euclidean Space (Proc. Sympos. Pure Math., WilliamsColl., Williamstown, Mass., 1978), Part 1. Proc. Sympos. Pure Math., vol. 35, pp. 163-165. Amer. Math. Soc., Providence (1979)

9. Fan, D., Guo, K., Pan, Y.: $L^{p}$ estimates for singular integrals associated to homogeneous surfaces. J. Reine Angew. Math. $542,1-22(2002)$

10. Fan, D., Pan, Y.: Singular integral operators with rough kernels supported by subvarieties. Am. J. Math. 119, 799-839 (1997)

11. Frazier, M., Jawerth, B., Weiss, G.: Littlewood-Paley Theory and the Study of Function Spaces. CBMS Reg. Conf. Ser., vol. 79. Am. Math. Soc., Providence (1991)

12. Grafakos, L., Stefanov, A.: $L^{p}$ bounds for singular integrals and maximal singular integrals with rough kernels. Indiana Univ. Math. J. 47, 455-469 (1998)

13. Honzik, P.: Examples of singular integral operators with rough kernels. Proc. Am. Math. Soc. (to appear)

14. Korry, S.: Boundedness of Hardy-Littlewood maximal operator in the framework of Lizorkin-Triebel spaces. Rev. Mat. Complut. 15, 401-416 (2002)

15. Liu, F:: Integral operators of Marcinkiewicz type on Triebel-Lizorkin spaces. Math. Nachr. 290, 75-96 (2017)

16. Liu, F: A note of Littlewood-Paley functions on Triebel-Lizorkin spaces. Bull. Korean Math. Soc. 55, 659-672 (2018)

17. Liu, F.: A note on Marcinkiewicz integrals associated to surfaces of revolution. J. Aust. Math. Soc. 104, 380-402 (2018)

18. Liu, F: Boundedness and continuity of maximal operators associated to polynomial compound curves on Triebel-Lizorkin spaces. Math. Inequal. Appl. 22, 25-44 (2019)

19. Liu, F., Mao, S., Wu, H.: On rough singular integrals related to homogeneous mappings. Collect. Math. 67, 113-132 (2016)

20. Liu, F., Wu, H.: On the regularity of maximal operators supported by submanifolds. J. Math. Anal. Appl. 453, 144-158 (2017)

21. Liu, F., Xue, Q., Yabuta, K.: Rough maximal singular integral and maximal operators supported by subvarieties on Triebel-Lizorkin spaces. Nonlinear Anal. TMA 171, 41-72 (2018)

22. Liu, F., Xue, Q., Yabuta, K.: Boundedness and continuity of maximal singular integrals and maximal functions on Triebel-Lizorkin spaces. Sci. China Math. 63, 907-936 (2020)

23. Ricci, F., Stein, E.M.: Harmonic analysis on nilpotent groups and singular integrals I: osicllatory integrals. J. Funct. Anal. 73, 179-194 (1987)

24. Sato, S.: Estimates for singular integrals and extrapolation. Stud. Math. 192, 219-233 (2009)

25. Triebel, H.: Theory of Function Spaces. Monogr. Math., vol. 78. Birkhäser, Basel (1983)

26. Yabuta, K: Triebel-Lizorkin space boundedness of Marcinkiewicz integrals associated to surfaces. Appl. Math. J. Chin. Univ. 30, 418-446 (2015)

27. Zhang, C., Chen, J.: Boundedness of $g$-functions on Triebel-Lizorkin spaces. Taiwan. J. Math. 13, 973-981 (2009)

\section{Submit your manuscript to a SpringerOpen ${ }^{\circ}$ journal and benefit from:}

- Convenient online submission

- Rigorous peer review

- Open access: articles freely available online

- High visibility within the field

- Retaining the copyright to your article

Submit your next manuscript at $\gg$ springeropen.com 